\title{
Reconstrução de cartilagem tireoide com enxerto costocondral durante abordagem primária em trauma cervical penetrante - relato de caso
}

Thyroid cartilage reconstruction with costochondral graft during primary approach in penetrating cervical trauma - case report

Reconstrucción de cartílago tiroideo con injerto costocondral durante abordaje primario en trauma cervical penetrante - reporte de caso

\section{Resumo}

O trauma de laringe constitui-se em uma lesão penetrante na região cervical de grande importância nos atendimentos em emergências. Lesões penetrantes estão associadas a trauma balístico, com maior chance de ruptura endo laríngea. Este relato de caso refere-se a um paciente do sexo masculino vítima de lesão penetrante promovido por arma de fogo em região cervical anterior, com lesão laríngea complexa, grau IV de Legacy Emanuel. Este foi submetido a uma cervicotomia de emergência, com evidência de lesão de cartilagem tireoide, sendo realizado enxerto cartilaginoso proveniente de segunda articulação costocondral direita, tendo o paciente evoluído de maneira satisfatória em pós operatório sem déficits funcionais de fala. Foi evidenciado, que a aplicação da técnica resultou na não rejeição do órgão ao enxerto, com rápida recuperação. Houve uma completa epitelização de face intraluminal do enxerto no quinto dia pós-operatório, evidenciado em broncoscopia e resultando em ganho funcional e estético para o paciente, além de tempo de internação hospitalar reduzido. Esta estratégia adotada gerou bons resultados, sem rejeição de retalho, com boa repercussão estética e sem limitações funcionais no doente, reduzindo o número de abordagens cirúrgicas, tempo hospitalizado e gastos de recursos financeiros com internação hospitalar.

Palavras-chave: Ferimentos por arma de fogo; Broncoscopia; Traumatismos do nervo laríngeo; Traumatismos do pescoço; Ferimentos penetrantes.

\footnotetext{
Abstract

Laryngeal trauma is a penetrating injury in the cervical region with great importance in emergency care. Penetrating injuries are associated with ballistic trauma, with a greater chance of endolaryngeal rupture. This case report refers to a male patient who suffered a penetrating injury caused by a firearm in the anterior cervical region with a complex laryngeal lesion, grade IV of Legacy Emanuel. He underwent an emergency cervicotomy with evidence of thyroid cartilage injury, and a cartilaginous graft was performed from the second right costochondral joint, and the patient progressed satisfactorily in the postoperative period without functional speech deficits. It was demonstrated that the
} 
application of the technique resulted in the non-rejection of the organ to the graft, with rapid recovery. There was complete epithelialization of the intraluminal face of the graft on the fifth postoperative day evidenced by bronchoscopy and resulting in functional and aesthetic gain for the patient, in addition to a shorter hospital stay. This adopted strategy generated good results without flap rejection, with good aesthetic repercussions and without functional limitations in the patient, reducing the number of surgical approaches, hospitalized time and expenditure of financial resources with hospitalization.

Keywords: Gunshot wounds; Bronchoscopy; Laryngeal nerve injuries; Neck injuries; Penetrating wounds.

\section{Resumen}

El traumatismo laríngeo es una lesión penetrante en la región cervical de gran importancia en la atención de urgencias. Las lesiones penetrantes se asocian con trauma balístico, con mayor probabilidad de ruptura endolaríngea. Este reporte de caso se refiere a un paciente masculino que sufrió una lesión penetrante por arma de fuego en la región cervical anterior, con una lesión laríngea compleja, grado IV de Legacy Emanuel. Se le realizó cervicotomía de emergencia, con evidencia de lesión del cartílago tiroides, y se realizó injerto cartilaginoso de la segunda articulación costocondral derecha, y el paciente evolucionó satisfactoriamente en el postoperatorio sin déficits funcionales del habla. Se evidenció que la aplicación de la técnica resultó en el no rechazo del órgano al injerto, con rápida recuperación. Hubo epitelización completa de la cara intraluminal del injerto al quinto día postoperatorio, evidenciada por broncoscopia, lo que resultó en una ganancia funcional y estética para el paciente, además de una menor estancia hospilaria. Esta estrategia adoptada generó buenos resultados, sin rechazo del colgajo, con buena repercusión estética y sin limitaciones funcionales en el paciente, reduciendo el número de abordajes quirúrgicos, el tiempo de hospitalización y el gasto de recursos económicos con la hospitalización.

Palabras clave: Heridas de bala; Broncoscopia; Lesiones del nervio laríngeo; Lesiones en el cuello; Heridas penetrantes.

\section{Introdução}

Situada entre a faringe e a traqueia e localizada na porção cervical anterior, a laringe é uma estrutura tubular compreendida por cartilagens que são interligadas através de ligamentos, mobilizadas por músculos que formam articulações e desempenha funções respiratória, fonatória e esfincteriana; apresentando relações anatômicas com glândulas endócrinas, trato gastrointestinal, estruturas vasculares, ósseas e nervosas (Pérez Alcantud et al., 2020; Demetriades et al., 2001). Mesmo com sua mobilidade e capacidade de se deslocar quando pressionada ou atingida, devido sua localização mais superficial e ausência de anteparo ósseo, a laringe torna-se vulnerável a traumas externos (Pérez Alcantud et al., 2020).

As lesões laríngeas ocorrem mais comumente em decorrência de mecanismos iatrogênicos, principalmente relacionada a longos períodos de intubação orotraqueal, este correspondendo a aproximadamente 90\% dos mecanismos de lesão endolaríngea, seguidos de traumas contusos, traumas penetrantes e inalação de substâncias cáusticas (Benjamin, 2018; Farley, et al., 2021; Pérez Alcantud et al., 2020; Reid \& Ha, 2019). Em todas estas situações, o prognóstico do paciente está intimamente ligado ao momento do diagnóstico da lesão, quando mais cedo identificada e tratada, menores as chances do paciente evoluir com comprometimento funcional da estrutura, ou mesmo com oclusão de via aérea pelo edema local em decorrência do trauma (Welter, et al., 2020; Farley, et al., 2021; Sachdeva \& Upadhyay, 2017)

Desta forma, ter ciência do mecanismo de trauma, realizar exame físico criterioso e deter conhecimento anatômico detalhado da região cervical, além de acesso a exames complementares direcionados, é de extrema importância a fim de detectar lesões precocemente (Pérez Alcantud et al., 2020; Herrera, et al. 2020, Elias et al., 2021; Ford et al., 1995)

O trauma de laringe está presente em 18\% das lesões penetrantes na região cervical (Jewett et al., 1999; Elias et al., 2021), correspondendo a apenas um em 5.000 a 137.000 atendimentos em emergências (Bent et al., 1993; Jewett et al., 1999; Elias et al., 2021). Destes, um em 445 pacientes com lesões laríngeas graves (Verschueren et al., 2006). Lesões penetrantes estão geralmente associadas a trauma balístico, com maior chance de ruptura endolaríngea (Elias et al., 2021). Embora não haja um algoritmo universal a ser seguido, a literatura mais atual utiliza as classificações de Legacy Emanuel e Schaefer-Fuhrman como base para determinar a gravidade da lesão laríngea e qual tratamento a ser proposto ao paciente (Madsen et al., 2016; Tabarcea, et al. 2020; Herrera, et al. 2020; Jewett et al., 1999; Elias et al., 2021, Ford et al., 1995) 
O objetivo deste trabalho é apresentar um relato de caso de um paciente com lesão laríngea complexa, grau IV de Legacy Emanuel e grau IV de Schaefer-Fuhrman, tratado com enxerto cirúrgico primário, além de realizar ampla revisão literária sobre o tema.

\section{Metodologia}

Este estudo é de natureza qualitativa com fundamentação metodológica científica (Ludke \& André et al., 1986; Pereira et al., 2018; Merriam et al., 1988; Yin et al., 2005) por meio de coleta de dados do paciente através do acesso ao prontuário eletrônico e exames complementares, sendo então reagrupados em apresentação cronológica para adequada compreensão do quadro e evolução clínica. Tais dados serão descritos em formato de caso clínico, o qual posteriormente será comparado com o conhecimento da literatura médica acerca do assunto.

As unidades assistenciais não foram identificadas a fim de preservar as instituições.

O paciente forneceu termo de consentimento livre e esclarecido para a publicação do caso e das imagens presentes nesse relato.

\section{Relato}

Paciente de 39 anos, sexo masculino foi admitido em box de emergência em Hospital Regional do Distrito Federal (DF); chegou por meios próprios, conduzindo o próprio veículo, com história de ferimento por arma de fogo há 15 minutos em região cervical anterior. Foi realizada a avaliação primária conforme protocolo preconizado pelo Advanced Trauma Life Suport (ATLS):

A: Estridor respiratório, fonação prejudicada, com rouquidão. Extensa lesão em zona 2 cervical, em topografia de cartilagem tireoide, com evidente perfuração e exposição de componentes intra-glóticos;

B: Murmúrio vesicular presente e simétrico sem ruídos adventícios, expansibilidade torácica simétrica, som claro pulmonar em todo o tórax, $\mathrm{SatO}_{2}$ 96\% em ar ambiente. Frequência respiratória: 30 irpm;

C: Sangramento ativo não pulsátil anterior, em zona 2 cervical. Pulsos periféricos presentes, tempo de enchimento capilar menor que 2 segundos, frequência cardíaca: 80 BPM;

D: Escala de Coma de Glasgow: 15, pupilas isocóricas e fotorreagentes; mobilidade dos quatro membros, com sensibilidade preservada;

E: Sem evidência de outras lesões em outras localidades, após exposição completa do paciente.

Foi provido suporte ventilatório com oxigênio suplementar por máscara não reinalante, com 15 litros por minuto, até garantia de via aérea definitiva provida em centro cirúrgico por equipe especializada. Após puncionados 2 acessos venosos periféricos calibrosos, paciente foi encaminhado para cervicotomia de emergência.

Durante a indução anestésica, o paciente apresentou quadro de bradicardia, revertida com atropina. Após estabilização, foi procedida a intubação orotraqueal por sequência rápida, bem sucedida na primeira tentativa, por equipe de anestesiologia.

A realização de uma cervicotomia de emergência evidenciou lesão cervical contendo destruição de parede de cartilagem tireoide anterior e porção membranosa (Figura 1). Foi realizada traqueostomia abaixo da lesão, a nível de $3^{\circ}$ anel traqueal para garantia da via aérea, com tubo número 6,5 (Figura 1). Durante o ato operatório, foi realizada a rafia de porção membranosa glótica, com fio de poliglactina 4.0 e desbridamento de bordas da cartilagem tireóide. 
Figura 1. Visão frontal de laceração promovida pelo projétil, com exposição da lesão da cartilagem tireóide. Traqueostomia realizada abaixo do terceiro anel traqueal.

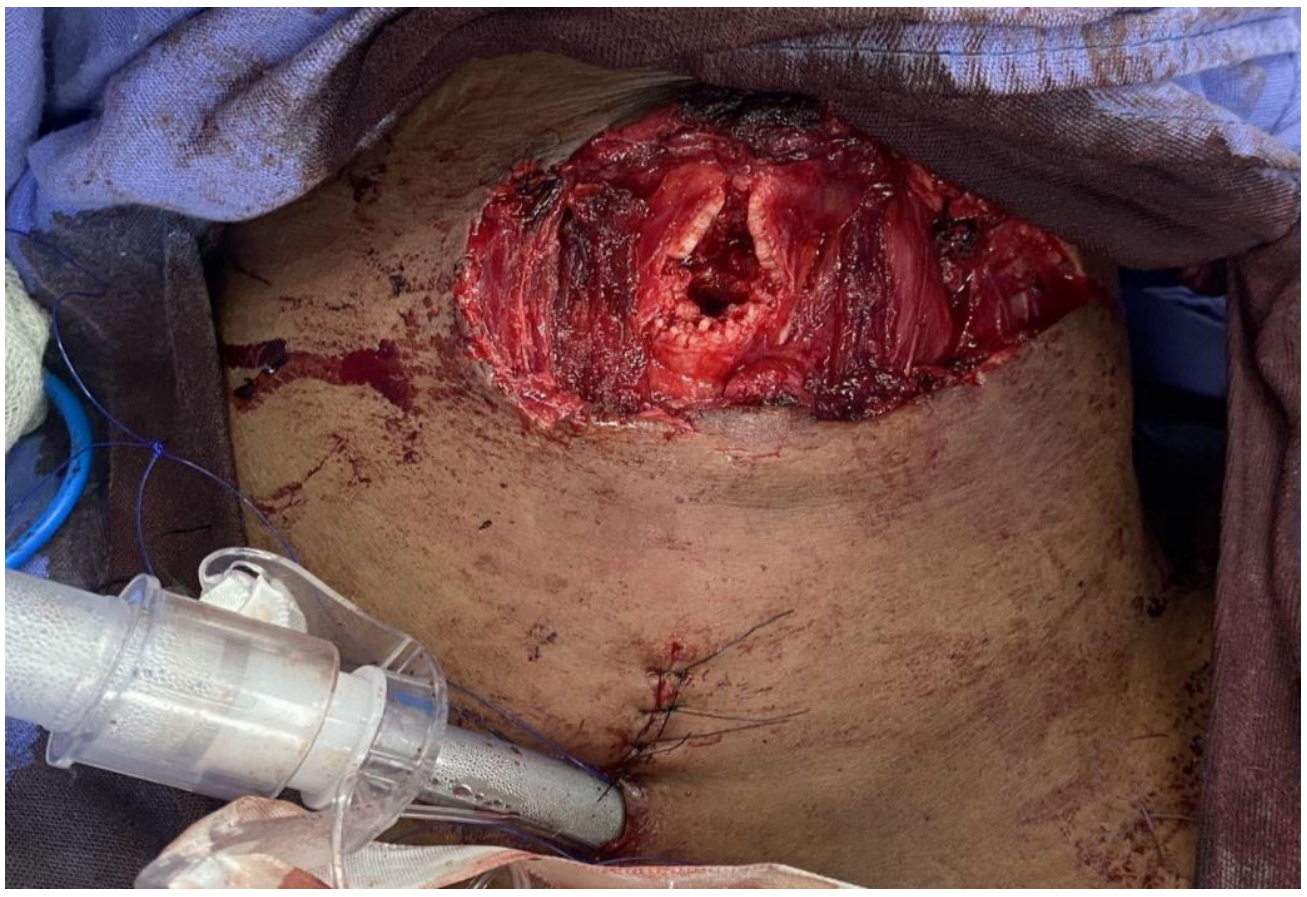

Fonte: Autores.

Realizou-se incisão à Chamberlain, em parede torácica anterior à direita; o pericôndrio foi removido e efetuada excisão parcial de porção cartilaginosa de $2^{\circ}$ arco costal (Figura 2).

Figura 2. Incisão à Chamberlain, com exposição de seguimento condral a ser retirado.

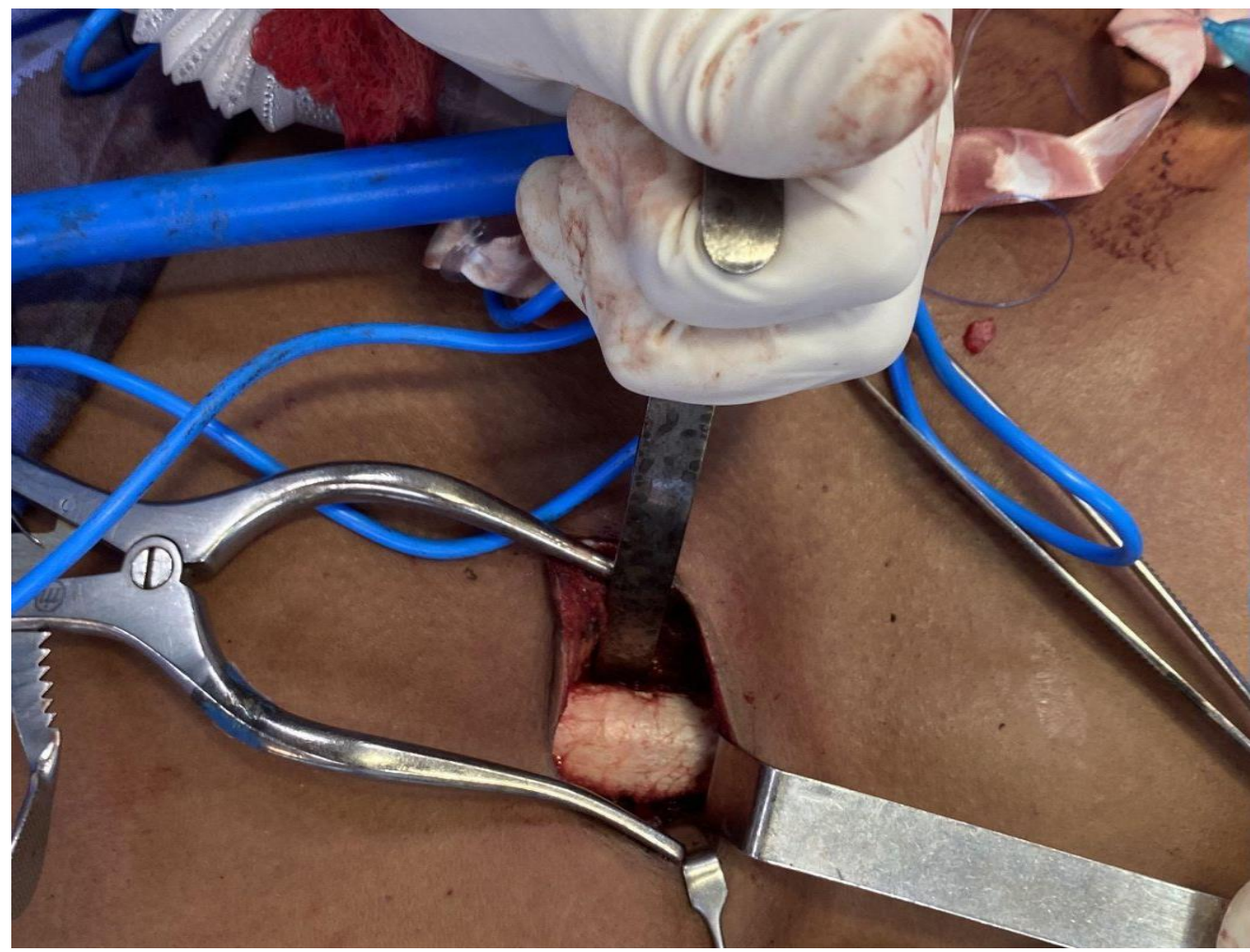

Fonte: Autores. 
Foram obtidas medidas baseadas em área destruída de cartilagem tireoide anterior, moldado cartilagem extraída de segundo espaço intercostal (Figura 3) conforme medidas e confeccionado enxerto local fixando-o com fios de polidioxanona e poliglecaprone 3-0, com pontos em X (Figura 4).

Figura 3. Molde e enxerto cartilaginoso utilizado.

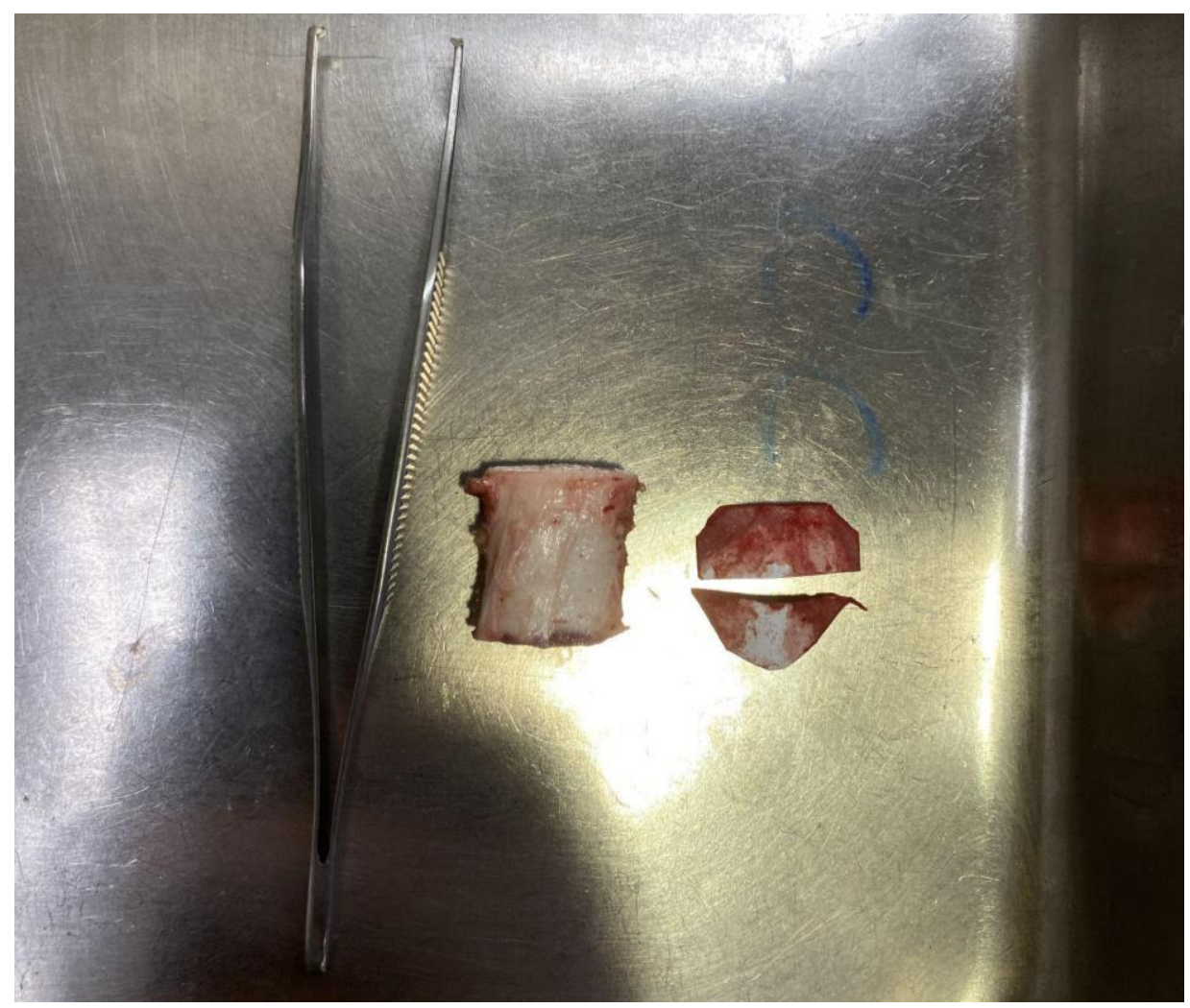

Fonte: Autores.

Figura 4. Resultado final de enxerto cartilaginoso.

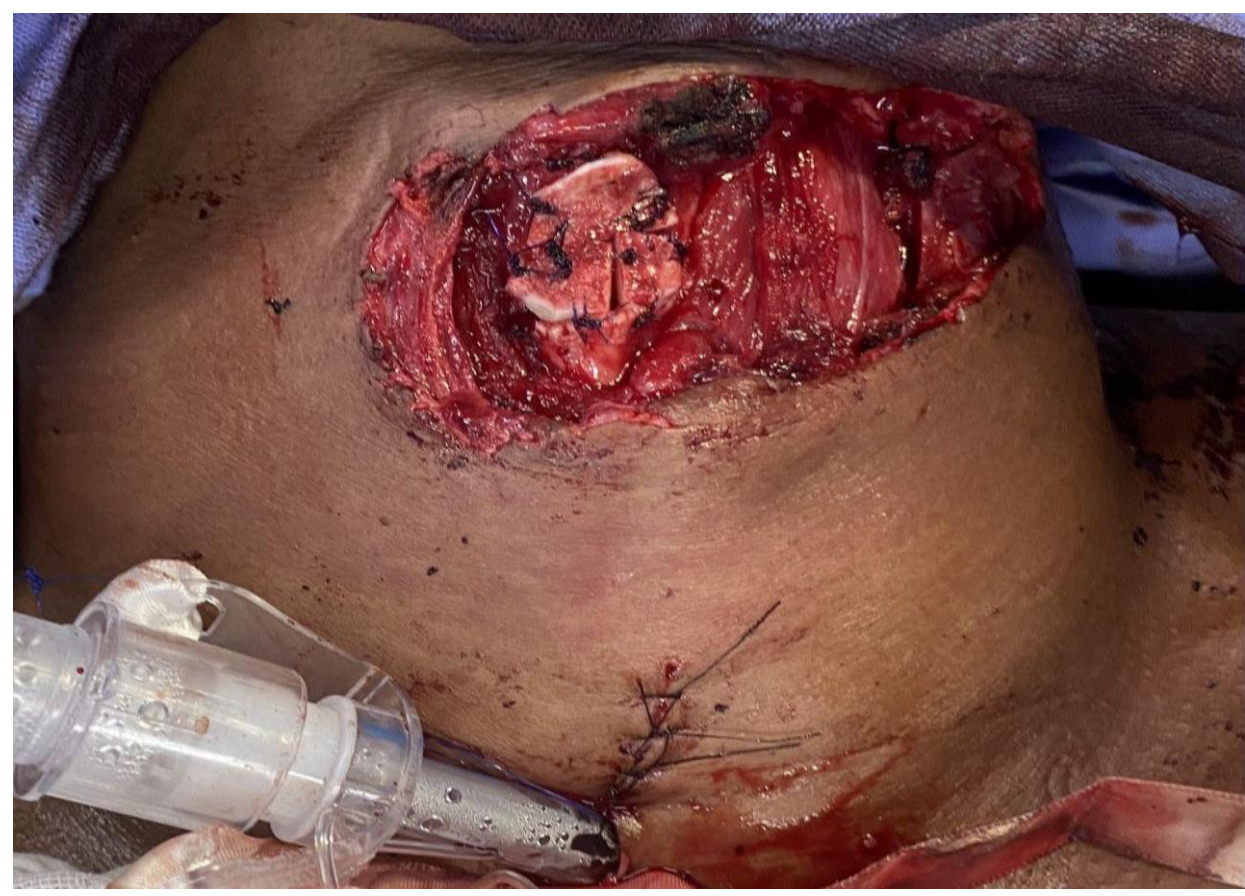

Fonte: Autores. 
Realizou-se patch com musculatura de esterno-hióideo utilizando fio de poliglactina 3-0, com pontos em X, sobre região de reparo cartilaginoso. Não foram evidenciadas lesões vasculares ou esofágicas durante a cervicotomia. A hemostasia foi revisada e foi feita a síntese dos tecidos por planos. Após término do procedimento, em pósoperatório imediato, não foi evidenciado escape aéreo por ferida operatória ou surgimento de enfisema subcutâneo. No pósoperatório foi prescrita antibioticoterapia com ampicilina e sulbactam por dez dias, corticoterapia, sintomáticos.

Paciente foi avaliado pela equipe de fonoaudiologia da unidade no segundo dia pós-operatório, a qual constatou recuperação na capacidade de fonação sem aparente perda funcional da movimentação das cordas vocais, à despeito do edema local no pós-operatório, através de válvula de fonação em traqueostomia, sendo também, permitida a reintrodução da alimentação via oral, com progressão de consistência gradativa, após ausência de distúrbios de deglutição evidenciado pela equipe.

No $5^{\circ}$ dia pós-operatório foi realizada broncoscopia, na qual foi evidenciada epitelização de toda porção intra-luminal, que compreendia o enxerto cartilaginoso, além de hematoma na face laríngea da epiglote e hipertrofia das pregas ventriculares (Figura 5). Não foram evidenciadas lesões de continuidade da traquéia. Realizou-se a troca de cânula de traqueostomia, por cânula metálica, número 7,0 .

Figura 5. Broncoscopia no quinto dia pós-operatório.

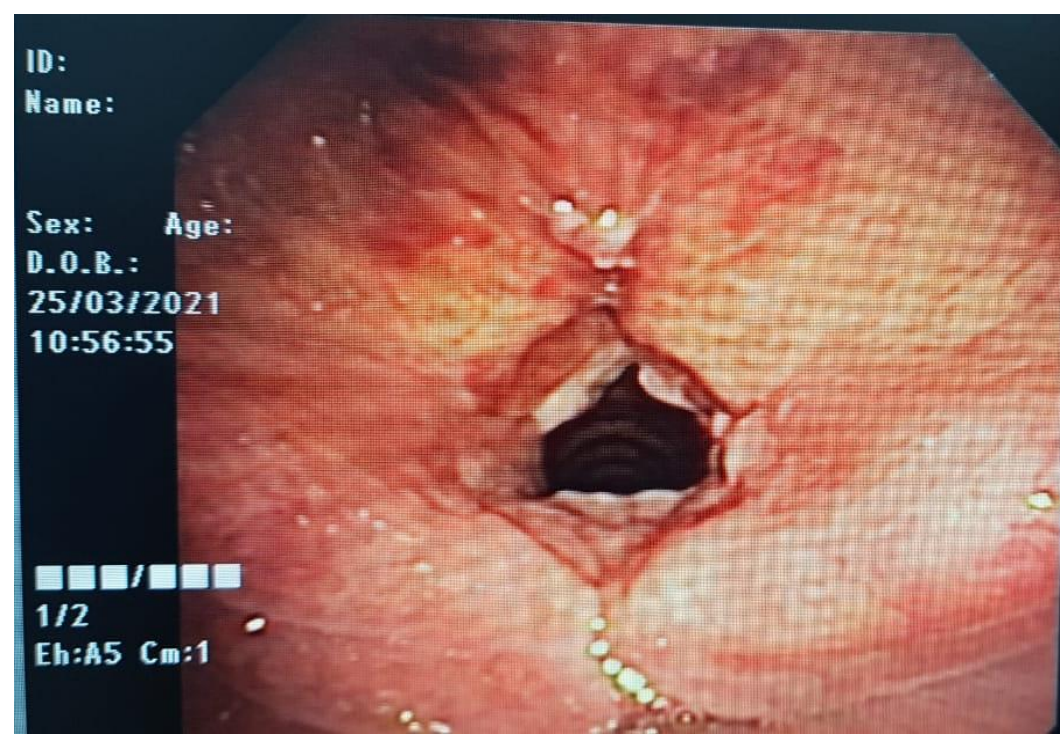

Fonte: Autores.

O paciente recebeu alta hospitalar no $8^{\circ}$ dia pós-operatório, com manutenção do uso de amoxicilina + ácido clavulânico (500 mg + $125 \mathrm{mg}, 8 / 8$ horas), até completar dez dias de terapia, além de prednisona 10 miligramas por 10 dias.

\section{Discussão}

A intubação orotraqueal é a via aérea avançada preferida durante primeiro contato e estabilização do doente, em situações com evidência de que a pervidade da via aérea está comprometida. Porém, este procedimento pode oferecer riscos como piora da lesão traumática e formação de falso trajeto em via aérea. Desta forma, tal procedimento deve ser realizado por equipe experiente, de preferência equipe de anestesiologia, tendo sempre à disposição equipe capacitada para realização de via aérea cirúrgica (Elias et al., 2021; Schaefer, 2014; Krausz et al., 2015).

Conforme a classificação de Legacy Emanuel, para trauma laríngeo, lesões classificadas em grau II ou mais, apresentam indicação formal de proteção de via aérea através de traqueostomia, ainda em abordagem cirúrgica primária, devido risco de oclusão de via aérea, proporcionada pelo edema na região traumatizada nas primeiras horas ou dias de pós trauma (Elias et al., 
2021). Estes mesmo autores também ponderam que, embora a cricotireoidostomia cirúrgica seja preferida em cenários de emergência ou urgência, nesta situação ela interfere na reconstrução laríngea, podendo inclusive contribuir para limitação da movimentação cricotireóide, limitando amplitude vocal.

Os estudos dispostos na literatura mais atual mostram que em situações em que há lesão endolaríngea com envolvimento de região glótica, o diagnóstico precoce e intervenção precoce, em até 24 a 48 horas, gera resultados superiores na prevenção de sequelas funcionais ao paciente; entre elas, a menor chance de formação de sinéquias em cordas vocais, diminuindo assim perda funcional da fala e principalmente permeabilidade das vias aéreas (Elias et al., 2021; Schaefer, 2014; Sachdeva \& Upadhyay, 2017).

O enxerto com cartilagem costocondral já é utilizado em uma gama de procedimentos estéticos eletivos. Entretanto, no cenário do trauma sua aplicabilidade ainda não é bem difundida. Em um estudo realizado pelo Departamento de Otorrinolaringologia e Cirurgia de Cabeça e Pescoço da Universidade da Carolina do Norte ficou evidenciado que pacientes vítimas de trauma laríngeo, com necessidade de abordagem cirúrgica, tiveram tempo médio de internação hospitalar de 13 dias, com os custos referentes à internação hospitalar de, aproximadamente, vinte e dois mil dólares (Jewett et al., 1999).

No presente relato de caso, o enxerto costocondral foi utilizado para a reconstrução da cartilagem tireóide, durante abordagem primária de lesão traumática penetrante, por arma de fogo. E, tal procedimento, não se encontra registrado na literatura.

Evidenciou-se, portanto, que a aplicação da técnica apresentada neste relato de caso resultou na não rejeição do órgão ao enxerto, além de uma rápida recuperação do doente e com completa epitelização de face intra-luminal do enxerto no quinto dia pós operatório; fato evidenciado em broncoscopia (Figura 5), resultando, desta forma, em ganho funcional e estético para o paciente (Figura 6), além de um tempo de internação hospitalar mais reduzido e, consequentemente, diminuindo gastos adicionais relacionados à internação.

Figura 6. Resultado estético final no terceiro dia pós-operatório.

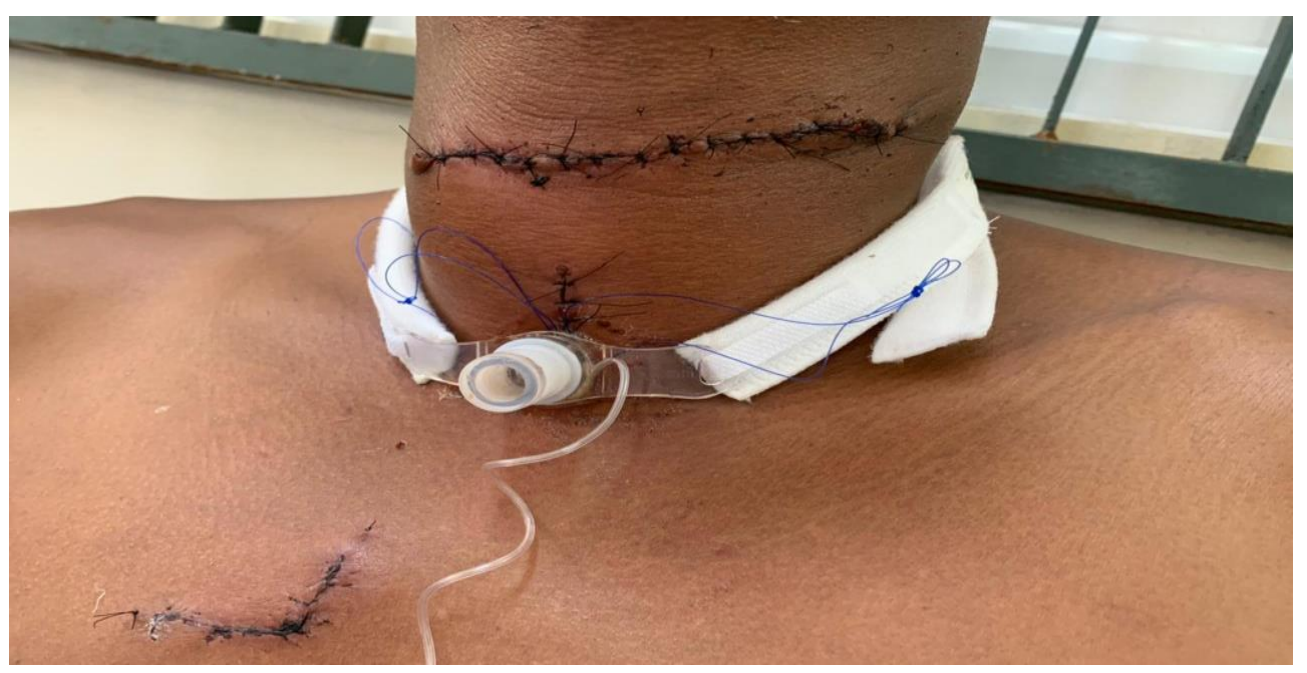

Fonte: Autores.

\section{Considerações Finais}

Neste relato conduziu-se um paciente com lesão traumática penetrante, provida por arma de fogo, admitido em unidade de atenção terciária com perviedade de via aérea comprometida, devido lesão transfixante da face anterior da cartilagem tireóide, apresentando sinais clínicos de obstrução da via aérea, como estridor respiratório, respiração superficial e taquipneia.

Durante exame físico admissional foi possível observar exposição e distorção de anatomia glótica. 
Optou-se em abordagem primária por realização de laringoplastia em cartilagem tireóide com enxerto costocondral de segunda costela à direita.

Houve evolução com boa reepitelização da face interna de região reconstruída no quinto dia pós-operatório, evoluindo sem déficit funcional de fala.

Tal estratégia adotada gerou bons resultados, sem rejeição de retalho, com significante resultado estético e sem limitações funcionais no paciente, reduzindo número de abordagens cirúrgicas, tempo de internação hospitalar e gastos de recursos financeiros com internação em ambiente hospitalar.

A literatura ainda é escassa e carece de estudos mais aprofundados sobre manejo por meio de reconstrução cirúrgica completa de laringe, em decorrência de trauma penetrante.

A publicação de estudos com a abordagem de tal tema poderá contribuir para a melhor compreensão e sistematização de abordagem terapêutica de pacientes vítimas de tal afecção contribuindo, assim, para o retorno precoce do paciente às suas atividades cotidianas.

É necessária a realização de um algoritmo universal com o intuito de padronizar condutas e abordagens relacionadas ao trauma de laringe.

\section{Notas}

Não há publicações ou envios anteriores com informações sobrepostas, incluindo estudos e pacientes.

Este relato de caso não recebeu nenhuma bolsa específica de agências de fomento de setores público, comercial ou sem fins lucrativos.

Os autores declaram que não têm conflitos de interesse a divulgar.

\section{Referências}

Benjamin, B. (2018). Prolonged Intubation Injuries of the Larynx: Endoscopic Diagnosis, Classification, And Treatment. Annals of Otology, Rhinology \& Laryngology, 127 (8): 492-507. https://doi.org/10.1177/0003489418790348

Bent J. P, Silver J. R, \& Porubsky, E. S. (1993). Acute laryngeal trauma: a review of 77 patients. Otolaryngology Head \& Neck Surgery, 109 (3): $441-9$.

Demetriades, D., Velmahos, G. G., \& Asensio, J. A. (2001). Cervical Pharyngoesophageal and Laryngotracheal Injuries. World Journal of Surgery, 25 (8): 1044-1048. https://doi.org/10.1007/s00268-001-0057-9

Elias, N., Thomas, J., Cheng, A. (2021). Management of Laryngeal Trauma. Oral and Maxillofacial Surgery Clinics of North America, 33 (3):417-427. 10.1016/j.coms.2021.04.007.

Farley, L. S., \& Schlicksup, K. E. (2021). Tracheal Injury. In StatPearls. StatPearls Publishing.

Ford, H. R., Gardner, M. J., \& Lynch, J. M. (1995). Laryngotracheal disruption from blunt pediatric neck injuries: Impact of early recognition and intervention on outcome. Journal of Pediatric Surgery, 30 (2): 331-335. https://doi.org/10.1016/0022-3468(95)90584-7

Herrera, M., Tintinago, L., Victoria, W., Ordoñez, C. A., Parra, M., Betancourt-Cajiao, M., Caicedo, Y., Guzman, M., Gallego, L., Gonzalez Hadad, A., Pino, L., Serna, J., García, A., Serna, C., \& Hernandez, F. (2020). Damage Control of Laryngotracheal Trauma: The Golden Day. Colombia Médica, 51(4): 1-9. https://doi.org/10.25100/cm.v51i4.4599

Jewett, B. S., Shockley, W. W., \& Rutledge, R. (1999). External laryngeal trauma analysis of 392 patients. Archives of Otolaryngology Head \& Neck Surgery Aug. 125 (8), 877-80. 10.1001/archotol.125.8.877.

Krausz, A. A., Krausz, M. M., \& Picetti, E. (2015). Maxillofacial and neck trauma: a damage control approach. World Journal of Emergency Surgery, WJES, 10(31):1-9. https://doi.org/10.1186/s13017-015-0022-9

Ludke, M., \& André, M. (1986). Pesquisa em educação: abordagens qualitativas. https://edisciplinas.usp.br/pluginfile.php/4091392/mod_resource/content /1/Lud_And_cap3.pdf

Madsen, A. S., Laing, G. L., Bruce, J. L., Oosthuizen, G. V., \& Clarke, D. L. (2016). An audit of penetrating neck injuries in a South African trauma service. Injury, 47 (1): 64-69. https://doi.org/10.1016/j.injury.2015.07.032

Merriam, S. B. (1988). Case study research in education: A qualitative approach. Jossey-Bass, 226p. 
Research, Society and Development, v. 11, n. 3, e1311326006, 2022

(CC BY 4.0) | ISSN 2525-3409 | DOI: http://dx.doi.org/10.33448/rsd-v11i3.26006

Pérez, A. R., Fuentes, E. L. \& Tamayo Bavastro, B. (2020). Trauma laríngeo abierto. Reporte de un caso. Multimed, 24 (2): $443-453$. http://www.revmultimed.sld.cu/index.php/mtm/article/view/1923

Reid, A., \& Ha, J. F. (2019). Inhalational injury and the larynx: A review. Burns, 45 (6), 1266-1274. https://doi.org/10.1016/j.burns.2018.10.025 Sachdeva, K., \& Upadhyay, A. (2017). Neck Trauma: ENT Prospects. Indian Journal of Otolaryngology and Head \& Neck Surgery, 69 (1): $52-57$. https://doi.org/10.1007/s12070-016-1048-3

Schaefer, S. D. (2014). Management of acute blunt and penetrating external laryngeal trauma. Laryngoscope, 124 (1): 233-44. 10.1002/lary.24068.

Tabarcea, L. (2020). Traumatismo da laringe. Trabalho Final do Curso de Mestrado Integrado em Medicina, Faculdade de Medicina, Universidade de Lisboa, 2020. http://hdl.handle.net/10451/46901

Verschueren, D. S., Bell, R. B., Bagheri, S. C., Dierks, E. J., Potter, B. E. Management of laryngo-tracheal injuries associated with craniomaxillofacial trauma. (2006). Journal of Oral Maxillofacial Surgery, 64 (2): 203-14. 10.1016/j.joms.2005.10.034.

Welter, S., \& Essaleh, W. (2020). Management of tracheobronchial injuries. Journal of Thoracic Disease, 12 (10): 6143-6151. https://doi.org/10.21037/jtd2019-as-05

Yin, R. K. (2015). Estudo de caso: planejamento e métodos. Bookman, 205p. 